\title{
Energy-Efficient Scheduling for Downlink Multi-User MIMO
}

\author{
Lingjia Liu †, Guowang Miao $\ddagger$, and Jianzhong(Charlie) Zhang $\S$ \\ $\dagger$ Department of Electrical Engineering and Computer Science, University of Kansas, USA. \\ $\ddagger$ School of Information and Communication Technology, KTH, the Royal Institute of Technology, Sweden. \\ $\S$ Dallas Technology Laboratory, Samsung Telecomm. America, USA.
}

\begin{abstract}
Multi-user MIMO is the enabling technology for LTE-Advanced systems to meet IMT-Advanced targets. The gain of multi-user MIMO is achieved partially through advanced usergrouping, user-scheduling, and precoding. Traditionally, multiuser MIMO scheduling focuses solely on spectral-efficiency [1]. That is, the scheduler will strike to balance the cell-edge user spectral-efficiency as well as the cell-average spectral-efficiency. Similar to spectral-efficiency, energy-efficiency is becoming increasingly important for wireless communications. The energy efficiency is measured by a classical measure, "throughput per Joule", while both RF transmit power and device electronic circuit power consumptions are considered. In this paper, an energyefficient proportional-fair scheduling is proposed for downlink multi-user MIMO systems. To specific, the scheduling algorithm is proposed to balance cell-edge energy-efficiency and the cellaverage energy-efficiency. The energy-efficient proportional-fair metric is defined and the optimal power allocation maximizing the performance measure is identified. System level evaluation suggests that multi-user MIMO could improve the energyefficiency of a wireless communication system significantly.

Index Terms: energy-efficiency, proportional-fair scheduling, multi-user MIMO, IMT-Advanced,power allocation.
\end{abstract}

\section{INTRODUCTION}

Next generation wireless communication systems, named International Mobile Telecommunications-Advanced (IMTAdvanced) systems, target to achieve another major advancement from the current $3 \mathrm{G}$ systems, in terms of achieving 1 Gbps for downlink spectral-efficiency and $500 \mathrm{Mbps}$ for uplink spectral-efficiency [2]. The spectral-efficiency requirements of the IMT-Advanced systems are specified into two performance measures: cell-average spectral-efficiency and cell-edge user spectral-efficiency. The cell-average spectral-efficiency specifies the average spectral-efficiency over all the active mobile stations (MS) present in a system and the cell-edge user spectral-efficiency is defined to be the 5\%ile of the spectralefficiency of the corresponding mobile stations. In order for a technology to be classified as IMT-Advanced technology, the cell-edge user spectral-efficiency target and the cell-average spectral-efficiency target have to be met simultaneously. This is challenging because there is usually a trade-off between celledge performance and cell-average performance. Therefore, a scheduler performing a good balance between these two performance measures is crucial for IMT-Advanced systems.

While the recently finalized 3GPP LTE standard allows us to achieve $300 \mathrm{Mbps}$ for downlink and $75 \mathrm{Mbps}$ for uplink with the introduction of orthogonal frequency divi- sion multiplexing (OFDM), and single-user multiple-inputmultiple-output (MIMO) techniques with spectrum reuse one, the downlink spectral-efficiency targets of the IMT-Advanced are not satisfied with existing technologies [3]. This is the major motivation to develop LTE-Advanced technology. As one of the key enabling technologies for LTE-Advanced systems, downlink multi-user MIMO is proposed to enhance the system performance [4]. By creating different spatial signals to multiple mobile stations in the downlink, MU-MIMO could help to improve both the cell-edge user spectral-efficiency as well as the average-cell spectral-efficiency partially through multi-user diversity. However, the introduction of multi-user MIMO also brings additional complexity at the base station to perform multi-user scheduling to trade-off the cell-edge performance and cell-average performance.

In addition to spectral-efficiency improvement, energyefficiency is becoming increasingly important for wireless communications because of the slow progress of battery technology [5] and the growing requirements of anytime and anywhere multimedia applications. With sufficient battery power, link adaptation can be geared toward peak performance delivery. However, with limited battery capacity, link adaptation could be adapted toward energy conservation to minimize battery drain. In this case, energy-efficient communication may also have the desirable benefits of reducing interference to mobile stations as well as lessening environmental impacts, e.g. heat dissipation and electronic pollution. Recently, there are many progress in energy-efficient communication schemes [6], [7], [8], [9], [10]. It is shown in [6] that when the transmission bandwidth approaches infinity, the minimum received signal energy per bit for reliable communication over additive white Gaussian noise (AWGN) channels, approaches $-1.59 \mathrm{~dB}$. For band-limited transmission, the lowest order modulation should be used [7]. However, the investigation in [6], [7] does not account for additional circuit power consumed during transmission. Energy dissipation of both transmitter circuits and radio-frequency output is investigated in [8], where the modulation level is adapted to minimize the energy consumption according to the simulation observations. In [9], these ideas are extended to a detailed analysis of circuit and transmit powers for both adaptive multiple quadrature amplitude modulation (M-QAM) and multiple frequency shift keying (MFSK) in AWGN channels for short range energy-efficient communications. In [10], energy-efficient link adaptation is 
proposed for OFDM frequency-selective channels where the transmission power and the circuit power of a communication system are jointly considered for the optimization.

In this paper, we address energy-efficient multi-user scheduling for MU-MIMO systems. Both circuit power and transmission power are taking into account when designing the energy-efficient scheduling and power allocation. The proposed multi-user scheduler balances the cell-edge energyefficiency and cell-average energy-efficiency optimally under the generalized proportional-fair energy-efficiency metric. Based on the structure of the multi-user scheduler, an optimal power allocation scheme is proposed to balance the energy consumption of circuit operations and that of the RF transmissions. We demonstrate the existence of a unique globally optimal power allocation strategy and analyze the characteristics of the scheme.

An outline of the paper is as follows. In Section II we present the system model. In Section III, the generalized energy-efficient proportional-fair metric is introduced and the intuition behind the metric is discussed. Furthermore, the energy-efficient scheduler for downlink MU-MIMO is proposed. In Section IV, the optimal power allocation scheme to maximize the energy-efficient proportional-fair metric is characterized. System level evaluation of the corresponding energy-efficient scheduling algorithm for a downlink multiuser MIMO system is described in Section V. Section VI concludes the paper.

\section{SySTEM MODEL}

In this section, we introduce the system model of a multiuser MIMO system. Throughout the paper, denote matrices by capital boldface letters, vectors by lowercase boldface, and scalars by either upper or lowercase letters without boldface.

A typical system model of a multi-user MIMO system with $N$ mobile stations is illustrated in Fig. 1. In this paper,

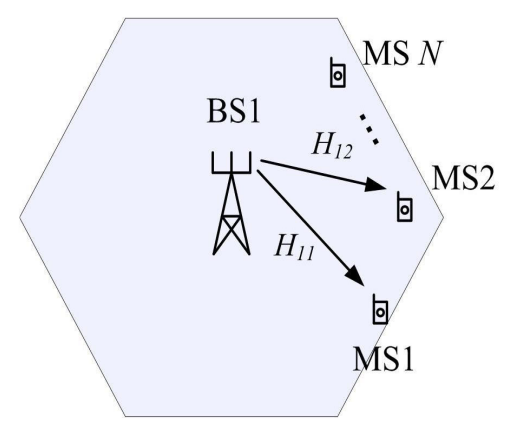

Fig. 1. Model of a multi-user MIMO System

we focus on a simple system where the base station is equipped with $N_{T}$ transmit antennas and the mobile stations are equipped with 1 receive antenna. Extension to multiple receive antennas follows similar methodology presented in the paper and can be treated as future work. In each time slot, the base station selects to serve $n$ mobile stations simultaneously among the $N$ mobile stations in a frequency resource, where $n \leq \min \left\{N, N_{T}\right\}$. Let $S_{n}$ be the set of selected MS indices. The received signal at each selected mobile station, say MS $i$ $\left(i \in S_{n}\right)$, can be expressed as

$$
y_{i}=\mathbf{h}_{1 i} \sum_{k \in S_{n}} \mathbf{f}_{k} \sqrt{p_{k}} x_{k}+n_{i}
$$

$x_{i}$ is the transmitted symbol of MS $i$ and $E\left[\left|x_{i}\right|^{2}\right]=1$, where $E$ is the expectation. $\sqrt{p_{i}}$ is the power allocation for MS $i$ 's data. $\mathbf{f}_{i}$ is the $N_{T} \times 1$ precoding/beam-forming vector for MS $i$ 's data. $\mathbf{h}_{1 i}$ denotes the $1 \times N_{T}$ channel matrix from the base station to MS $i . n_{i}$ is the interference-plus-noise vector at MS $i$, which is Gaussian distributed random variable with a mean of 0 and a variance of $\sigma^{2}$.

When $n>1$, the received signal at each mobile station suffers from the intra-cell interference; hence to take advantage of the spatial domain degrees of freedom, advanced precoding at the base station should be employed to mitigate the corresponding intra-cell interference. Accordingly, the received signals at MS $i$ in (1) can rewritten as

$$
y_{i}=\mathbf{h}_{1 i} \mathbf{f}_{i} \sqrt{p_{i}} x_{i}+\mathbf{h}_{1 i} \sum_{k \in S_{n}, k \neq i} \mathbf{f}_{k} \sqrt{p_{k}} x_{k}+n_{i} .
$$

where $\mathbf{h}_{1 i} \sum_{k \in S_{n}, k \neq i} \mathbf{f}_{k} \sqrt{p_{k}} x_{k}$ is the intra-cell interference for MS $i$. Let

$$
\begin{aligned}
\mathbf{H} & =\left[\mathbf{h}_{11}^{T} \ldots \mathbf{h}_{1 N}^{T}\right]^{T}, \\
\mathbf{F} & =\left[\mathbf{f}_{1} \ldots \mathbf{f}_{N}\right] .
\end{aligned}
$$

Here $[\cdot]^{T}$ is the transpose of a vector/matrix. When zeroforcing beam-forming is used at the base station for its simplicity, the precoding vectors of the selected mobile stations are chosen such that the multi-user intra-cell interference is zero, i.e. $\mathbf{h}_{1 i} \mathbf{f}_{k}=0$ for $i \neq k$. For a given selected MS indices set of $S_{n}$, let $\mathbf{H}\left(S_{n}\right)$ and $\mathbf{F}\left(S_{n}\right)$ be the corresponding submatrices of $\mathbf{H}$ and $\mathbf{F}$. The zero-forcing precoding/beam-forming vectors of $\mathbf{F}\left(S_{n}\right)$ that gives zero-interference is shown to be the pseudo inverse of $\mathbf{H}\left(S_{n}\right)$ [11] as

$$
\mathbf{F}\left(S_{n}\right)=\mathbf{H}\left(S_{n}\right)^{*}\left(\mathbf{H}\left(S_{n}\right) \mathbf{H}\left(S_{n}\right)^{*}\right)^{-1}
$$

Here $[\cdot]^{*}$ is the hermitian of a matrix.

From the zero-interference condition, the transmissions to the selected mobile stations are uncoupled. From (2), it can be seen that for MS $i, i \in S_{n}$, we have

$$
y_{i}=\sqrt{\gamma_{i} p_{i}} x_{i}+n_{i}
$$

where

$$
\gamma_{i}=\frac{1}{\left[\left(\mathbf{H}\left(S_{n}\right) \mathbf{H}\left(S_{n}\right)^{*}\right)^{-1}\right]_{i, i}},
$$

representing the effective channel power gain from the base station to MS $i$ after beam-forming/precoding. Denote $W$ as the system bandwidth, the achievable data transmission rate $r_{i}$ for MS $i$ can be expressed as [12]

$$
r_{i}=W \log \left(1+\frac{\gamma_{i} p_{i}}{\sigma^{2} \Gamma}\right)
$$


where $\Gamma$ is the signal-to-noise ration (SNR) gap that defines the gap between the channel capacity and a practical modulation and coding scheme (MCS). The SNR gap depends on the MCS used and the targeted error probability. For a coded quadrature amplitude modulation (QAM) system, the gap is given by

$$
\Gamma=9.8+\eta_{m}-\eta_{c}(\mathrm{~dB}),
$$

where $\eta_{m}$ is the system design margin and $\eta_{c}$ is the coding gain. For Shannon capacity, $\Gamma=0 \mathrm{~dB}$.

\section{ENERGY-EFFICIENT SCHEDULING}

As discussed in Section I, IMT-Advanced systems impose downlink spectral-efficiency requirements on both cell-average and cell-edge user simultaneously. In general, there is a tradeoff between these two performance measures. A scheduler biases towards cell-edge mobile stations will lead to a relatively large cell-edge spectral-efficiency with a low cellaverage spectral-efficiency. On the other hand, a scheduler biases towards cell-center mobile stations (such as opportunistic scheduler) will lead to a large cell-average spectralefficiency. As such, proportional-fair scheduling is widely used to achieve a good tradeoff of these two performance measures to meet the requirements simultaneously. Mathematically, the proportional-fair scheduling is trying to maximize the following utility function [13]

$$
f\left(T_{1}, \ldots, T_{N}\right)=\log T_{1}+\ldots+\log T_{N}=\sum_{k=1}^{N} \log T_{k}
$$

where $T_{i}$ stands for the accumulated throughput of MS $i$ in terms of bits. The log function is a monotonic increasing function, maximizing the utility function is equivalent to choose the scheduled mobile station which maximizes the rate of change of the corresponding utility function. Since spectralefficiency is defined to be "bit per second", the rate of change of the utility function should be taken with respect to time. Accordingly, when MS $i$ is scheduled, the rate of change with respect to time can be expressed as

$$
l_{s e i}=\frac{r_{i}}{T_{i}}
$$

where $l_{s e i}$ is the rate of change for spectral-efficiency, $r_{i}$ is the rate (bps), and $T_{i}$ is the accumulated throughput (bits) of MS $i$. As such, $r_{i} / T_{i}$ can be defined as the spectralefficiency proportional-fair metric for MS $i$. Accordingly, the proportional-fair scheduler of a single-user system will schedule the mobile station which has the highest proportionalfair metric:

$$
\arg \max _{i \in[1, \ldots, N]} \frac{r_{i}}{T_{i}}
$$

Following the same philosophy, the proportional-fair scheduler of a multi-user system can be expressed as [1]

$$
\arg \max _{S_{n} \in \Omega} \sum_{i \in S_{n}} \frac{r_{i}}{T_{i}},
$$

where $\Omega$ is the collection of all possible MS subsets. Note that finding the optimal MS subset is actually a NP-hard problem.
Since this scheduler is trying to balance the cell-edge spectralefficiency and the cell-average spectral-efficiency, we call it spectral-efficiency proportional-fair scheduler in this paper.

For energy-efficient communication, it is desirable to maximize the amount of data sent within a given amount of energy constraint [6] instead of the time constraint. As suggested in [10], a mobile station incurs additional circuit power during transmissions in addition to transmit power. Assuming the circuit power is relatively independent of the transmission rate as suggested in [8], the overall power consumption of serving MS $i$ at the base station, $p_{o}\left(r_{i}\right)$, can be expressed as

$$
p_{o}\left(r_{i}\right)=p_{C}+p_{T i}\left(r_{i}\right)
$$

where $p_{C}$ is the circuit power, $r_{i}$ is the achievable data rate in (3), and $p_{T i}\left(r_{i}\right)$ is the transmit power of $\mathrm{MS} i . p_{T i}\left(r_{i}\right)$ can be expressed as

$$
p_{T i}=\frac{p_{i}}{\xi}
$$

where $\xi \in[0,1]$ is the power amplifier efficiency and depends on the design and implementation of the transmitter.

Accordingly, given any amount of energy $e$, for MS $i$ the base station wants to maximize the energy-efficiency [10] as

$$
U\left(r_{i}\right)=\frac{T_{i}}{e} .
$$

$U(r)$ is called the energy-efficiency which represents how much data (bits) can be transmitted through a unit of energy (Joule). Maximizing the energy-efficiency for MS $i$ is equivalent to maximizing the amount of data sent to MS $i$ given a fixed amount of energy. As in the case of spectralefficiency, different mobile stations will have different energyefficiencies. Therefore, it is desirable to have uniform energyefficiency across the whole network just as the spectralefficiency requirements imposed by IMT-Advanced. Accordingly, we derive the energy-efficient proportional-fair scheduling as in the case of proportional-fair scheduling for spectralefficiency. Since the unit of the energy-efficiency is "bit per Joule", we want to maximize the utility function in (4) with respect to energy instead of time.

Assuming MS $i$ is being selected, the amount of energy $\Delta e$ consumed in a small duration, $\Delta t$, is

$$
\Delta e=p_{o}\left(r_{i}\right) \Delta t=\left(p_{C}+p_{T i}\right) \Delta t .
$$

The above equation assumes that the transmission power from the base station to MS $i$ does not change during $\Delta t$. Accordingly, the rate of change of the utility function with respect to energy when MS $i$ is scheduled can be expressed as

$$
l_{e e i}=\frac{1}{T_{i}} \frac{d\left(T_{i}\right)}{d(\Delta e)}
$$

where $l_{e e i}$ stands for the rate of change for energy-efficiency and $d(\cdot)$ represents the derivative operation. Taking (9) into account, the rate of change of the utility function with respect to energy can be expressed as

$$
l_{e e i}=\frac{1}{T_{i}} \frac{r_{i}}{p_{C}+p_{T i}}=\frac{1}{p_{C}+p_{T i}} \frac{r_{i}}{T_{i}}=\frac{l_{s e i}}{p_{C}+p_{T i}} .
$$


Similarly, we call $l_{e e i}$ the energy-efficient proportional-fair metric for MS $i$. It can be seen from (10) that the proportionalfair metric for energy-efficiency is different from that for spectral-efficiency and is inversely proportional to the overall power consumption. Furthermore, the following observations can be made for the relationship between $l_{s e}$ and $l_{e e}$ for a particular mobile station:

- increasing the transmission power will result in an increase in $l_{s e}$, however, it may cause a reduction in $l_{e e}$;

- reducing the transmission power will result in a reduction in $l_{s e}$, however, it may increase the corresponding $l_{e e}$;

- both proportional-fair metrics tend to penalize mobile stations which have been served for a relatively long time.

Accordingly, the energy-efficient proportional-fair scheduler of a single-user system will schedule the mobile station which has the highest energy-efficient proportional-fair metric:

$$
\arg \max _{i \in[1, \ldots, N]} \frac{1}{T_{i}} \frac{r_{i}}{p_{C}+p_{T i}} .
$$

When the circuit power is dominant, i.e. $p_{T i}<<p_{C}$, the energy-efficient proportional-fair scheduler becomes

$$
\arg \max _{i \in[1, \ldots, N]} \frac{1}{T_{i}} \frac{r_{i}}{p_{C}+p_{T i}} \approx \arg \max _{i \in[1, \ldots, N]} \frac{1}{p_{C}} \frac{r_{i}}{T_{i}},
$$

which is equivalent to the spectral-efficient proportional-fair scheduler shown in (6). For a multi-user system, similar method can be conducted to obtain the following energyefficient proportional-fair scheduler:

$$
\arg \max _{S_{n} \in \Omega} \sum_{i \in S_{n}} \frac{1}{T_{i}} \frac{r_{i}}{p_{C}+p_{T i}},
$$

\section{Power Allocation for Energy-EFFicient SCHEDULING}

In this section, we derive the optimal power allocation. To be specific, we demonstrate that a unique globally optimal power allocation always exists and provide the sufficient and necessary conditions for a power allocation scheme to maximize its energy-efficient proportional-fair metric.

It can be seen in (6), a base station should always use its maximum transmission power for MS $i$ in order to maximize the corresponding spectral-efficiency proportional-fair metric. However, this strategy is not true any more in the case of energy-efficient scheduling since transmission power is penalized in the denominator of the metric in (10). Furthermore, as seen in (10), the optimal power allocation scheme for MS $i$ can be defined as

$$
p_{i}^{*}=\arg \max _{p_{i}} \frac{\xi W \log \left(1+\frac{\gamma_{i} p_{i}}{\sigma^{2} \Gamma}\right)}{\left(\xi p_{C}+p_{i}\right) T_{i}} .
$$

Let

$$
V\left(p_{i}\right)=\frac{\xi W \log \left(1+\frac{\gamma_{i} p_{i}}{\sigma^{2} \Gamma}\right)}{\left(\xi p_{C}+p_{i}\right) T_{i}} .
$$

Accordingly, $V(x)$ defines a function from $[0, p]$ to $[0,+\infty)$, where $p$ is the maximum transmission power at the base station. Recall that for any $x \in X$,

$$
Q(x) \equiv\{z \in X: V(z) \geq V(x)\}
$$

is the better set of $x$. Let $V(x)=\mu$, it is clear that $\mu \geq 0$. Accordingly, $Q(x)$ can be expressed as

$$
\begin{aligned}
Q(x) & =\{z \in[0, p]: V(z) \geq \mu\} \\
& =\left\{z \in[0, p]: \frac{\xi W \log \left(1+\frac{\gamma_{i} z}{\sigma^{2} \Gamma}\right)}{\left(\xi p_{C}+z\right) T_{i}} \geq \mu\right\} \\
& =\left\{z \in[0, p]: \mu\left(\xi p_{C}+z\right) T_{i}-\xi W \log \left(1+\frac{\gamma_{i} z}{\sigma^{2} \Gamma}\right) \leq 0\right\}
\end{aligned}
$$

It is clear to see that $Q(x)$ is strictly convex for any value of $\mu$. Therefore, $V(x)$ is strictly quasiconcave in $x$ [14]. For a strictly quasiconcave function, if a local maximum exits, it is also globally optimal [14]. Therefore, a uniquely global optimal power allocation always exits.

The local optimal of $V\left(p_{i}\right)$ can be obtained by setting the partial derivative of $V\left(p_{i}\right)$ with respect to $p_{i}$ to zero. That is,

$$
\left.\frac{\partial V\left(p_{i}\right)}{\partial p_{i}}\right|_{p_{i}=p_{i}^{*}}=0
$$

Since $V\left(p_{i}\right)$ is quansiconcave in $p_{i}$, the local optimum is also the global optimum. The partial derivative of $V\left(p_{i}\right)$ with respect to $p_{i}$ can be expressed as

$$
\begin{aligned}
\frac{\partial V\left(p_{i}\right)}{\partial p_{i}} & =\frac{\xi W}{T_{i}\left(p_{i}+\xi p_{C}\right)}\left(\frac{\gamma_{i}}{\sigma^{2} \Gamma+\gamma_{i} p_{i}}-\frac{\log \left(1+\frac{\gamma_{i}}{\sigma^{2} \Gamma} p_{i}\right)}{p_{i}+\xi p_{C}}\right) \\
& =\frac{\xi W}{T_{i}\left(p_{i}+\xi p_{C}\right)}\left(w_{1}\left(p_{i}\right)-w_{2}\left(p_{i}\right)\right) .
\end{aligned}
$$

Note that $w_{1}(0)>0$ and $w_{2}(0)=0$ when $p_{C} \neq 0$. Therefore, when circuit power is non-negligible the result suggests that it is always beneficial to send data to a targeted mobile station. This observation is different from the intuition obtained in [10] where the base station will mute on some subcarriers under certain conditions. This is because in [10] no scheduling aspect is considered where the optimization is done to maximize overall system energy-efficiency. However, in the context of multi-user scheduling, mobile station will need to maximize its energy-efficient proportional-fair metric to maximize the scheduling opportunity. Also this result suggests that in the presence of circuit power, the optimal operation regime for energy-efficiency is not low power regime any more.

Now, let us analyze the structure of the optimal power allocation strategy. When $p_{i}^{*} \in[0, p]$, the optimal power allocation for MS $i$ 's energy-efficient proportional-fair metric is the solution of

$$
\frac{\gamma_{i}}{\sigma^{2} \Gamma+\gamma_{i} p_{i}^{*}}=\frac{\log \left(1+\frac{\gamma_{i}}{\sigma^{2} \Gamma} p_{i}^{*}\right)}{p_{i}^{*}+\xi p_{C}} .
$$

Accordingly, the maximum energy-efficient proportional-fair metric for MS $i$ can be expressed as

$$
V^{*}=\frac{\xi W \log \left(1+\frac{\gamma_{i} p_{i}^{*}}{\sigma^{2} \Gamma}\right)}{\left(\xi p_{C}+p_{i}^{*}\right) T_{i}}
$$

Insert (13) to (14), we have

$$
V^{*}=\frac{\xi W}{T_{i}} \frac{\gamma_{i}}{\sigma^{2} \Gamma+\gamma_{i} p_{i}^{*}} .
$$


Therefore, the optimal power allocation can be rewritten as

$$
p_{i}^{*}=\frac{\xi W}{T_{i} V^{*}}-\frac{\sigma^{2} \Gamma}{\gamma_{i}} .
$$

(15) suggests that the optimal power allocation follows a water-filling structure where the water level is determined by the optimal energy-efficient proportional-fair metric and the effective channel power gain of $\gamma_{i}$. Furthermore, the result suggests that in energy-efficient scheduling, the transmission power to different mobile station will be different even when the circuit power consumption at the base station is the same. This is different from the spectral-efficient scheduling where maximum transmission power is shown to be optimal.

\section{Performance Evaluations}

In this section, we evaluate the proposed multi-user MIMO scheduling algorithms for a cellular network. System parameters used in the system level evaluation is listed in the following table. Note that in the ITU urban macro (UMa)

TABLE I

System LeVEl Simulation PARAMETERS

\begin{tabular}{|c|c|}
\hline Parameters & Values \\
\hline System bandwidth & $10 \mathrm{MHz}$ \\
\hline FFT size & 1024 \\
\hline Number of data subcarriers & 600 \\
\hline Resource block (RB) size & 36 subcarriers \\
\hline Antenna configuration & 4 Tx and 1 Rx antennas \\
\hline Antenna spacing at BS & $4 \times$ Wavelength \\
\hline Antenna spacing at MS & $0.5 \times$ Wavelength \\
\hline Channel model & ITU UMa [15] \\
\hline MIMO Receiver at MS & MMSE \\
\hline Precoding method & Frequency-selective precoding \\
\hline Control overhead & $35.6 \%$ \\
\hline Circuit power $p_{C}$ & $100 \mathrm{~mW}$ \\
\hline Power amplifier efficiency $\xi$ & 0.5 \\
\hline Number of MSs in a cell & 10 \\
\hline
\end{tabular}

model, both large scale fading and small scale fading are included. Furthermore, mobile stations are randomly dropped indoor (with relatively large penetration loss) or outdoor (with relatively small penetration loss) with some predetermined probability. In the system level evaluation, the channel state information is assumed to be available at the base station. Table II illustrates the performance gains of multi-user MIMO energy-efficient scheduling as a function of the number of scheduled mobile stations. The results suggest that the multi-

TABLE II

ENERGY-EFFICIENCY COMPARISON

\begin{tabular}{|l|c|l|l|}
\hline & SU-MIMO & $\begin{array}{l}\text { MU-MIMO } \\
\text { with 2 MS }\end{array}$ & $\begin{array}{l}\text { MU-MIMO } \\
\text { with 4 MS }\end{array}$ \\
\hline $\begin{array}{l}\text { Cell-average perfor- } \\
\text { mance(bits/Joule) }\end{array}$ & 3.23 & 3.98 & 4.52 \\
\hline $\begin{array}{l}\text { Cell-edge performance } \\
\text { (bits/Joule) }\end{array}$ & 0.083 & 0.115 & 0.152 \\
\hline
\end{tabular}

user MIMO could significantly improve the energy-efficiency of a communication system due to the multi-user diversity. Furthermore, the proposed scheduler could help to reduce the energy-efficiency gaps among mobile stations hence improving both cell-edge and cell-average performance.

\section{CONCLUSION}

Multi-user MIMO is one of the key enabling techniques for LTE-Advanced. One of the challenges of MU-MIMO lies in how to apply multi-user scheduling to balance cell-edge and cell-average performance.

In this paper, we proposed an optimal scheduling algorithm in the context of generalized energy-efficient proportional-fair metric. The proposed scheduler balances cell-edge energyefficiency as well as cell-average energy-efficiency for multiuser MIMO systems. Optimal power allocation scheme is derived for the generalized energy-efficiency proportionalfair metric. When the circuit power is non-negligible, the result suggests that always transmitting at a relatively large power seems to be beneficial. The optimal power allocation scheme follows a water-filling structure where the water level depends on the optimal value of the generalized energyefficient proportional-fair metric.

The system level simulation results suggest that the proposed scheduler for multi-user MIMO systems can significantly improve the cell-average energy-efficiency as well as the cell-edge energy-efficiency. We believe this is a promising scheduling algorithm for IMT-Advanced systems including both 3GPP LTE-Advanced and IEEE 802.16m.

\section{REFERENCES}

[1] L. Liu, Y.-H. Nam, and J. Zhang, "Proportional fair scheduling for multicell multi-user MIMO systems," in Forty-Fourth Annual Conference on Information Sciences and Systems (CISS), 2010, pp. $1-6$.

[2] NTT DoCoMo, Update of E-UTRA and IMT-Advanced Requirements, January 2009.

[3] 3GPP, "RP-090939, 3GPP submission package for IMT-Advanced," in $3 G P P$ 45th Radio Access Network (RAN) Technical Specification Group (TSG) plenary meeting, September 2009.

[4] H. Weingarten, Y. Steinberg, and S. Shamai, "The capacity region of the gaussian multiple-input multiple-output broadcast channel," IEEE Transactions on Information Theory, vol. 52, no. 9, pp. 3936-3964, September 2006.

[5] K. Lahiri, A. Raghunathan, S. Dey, and D. Panigrahi, "Battery-driven system design: A new frontier in low power design," in International Conference on VLSI Design. IEEE, January 2002, pp. 261-267.

[6] S. Verdu, "Spectral efficiency in the wideband regime," IEEE Transactions on Information Theory, vol. 48, no. 6, pp. 1319-1343, June 2002.

[7] F. Meshkati, H. V. Poor, S. C. Schwartz, and N. B. Mandayam, "An energy-efficient approach to power control and receiver design in wireless networks," IEEE Transactions on Communications, vol. 5, no. 1, pp. 3306-3315, November 2006.

[8] A. Y. Wang, S. Cho, C. G. Sodini, and A. P. Chandrakasan, "Energy efficient modulation and mac for asymmetric RF microsensor system," in IEEE International Symposium on Low Power Electronics and Design, pp. 106-111, August 2001.

[9] S. Cui, A. J. Goldsmith, and A. Bahai, "Energy-constrained modulation optimization," IEEE Transactions on Wireless Communications, vol. 4, no. 5, pp. 2349-2360, September 2005.

[10] G. Miao, N. Himayat, and G. Y. Li, "Energy-efficient link adaptation in frequency-selective channels," IEEE Transactions on Communications, vol. 58, no. 2, pp. 545-554, February 2010.

[11] T. Yoo and A. Goldsmith, "On the optimality of multi-antenna broadcast scheduling using zero-forcing beamforming," IEEE Journal on Selected Areas in Communications, vol. 24, no. 3, pp. 528-541, March 2006.

[12] J. M. Cioffi, A Multicarrier Primer, ANSI T1E1.

[13] D. Tse and P. Viswanath, Fundamentals of Wireless Communication, Cambridge University Press, 2005.

[14] E. Wolfstetter, Topics in Microeconomics: Industrial Organization, Auctions, and Incentives, Cambridge University Press, 1999.

[15] ITU, Report M.2135: "Guidelines for evaluation of radio interface technologies for IMT-Advanced”, 2008. 